$11-3-2014$

\title{
Recurrent Aphthous Stomatitis: Diagnosis and Management in Primary Care
}

Jillian Hudson

Follow this and additional works at: https://aah.org/jpcrr

Part of the Immune System Diseases Commons, and the Primary Care Commons

\section{Recommended Citation}

Hudson J. Recurrent aphthous stomatitis: diagnosis and management in primary care. J Patient Cent Res Rev. 2014;1:197-200. doi: 10.17294/2330-0698.1040

Published quarterly by Midwest-based health system Advocate Aurora Health and indexed in PubMed Central, the Journal of Patient-Centered Research and Reviews (JPCRR) is an open access, peer-reviewed medical journal focused on disseminating scholarly works devoted to improving patient-centered care practices, health outcomes, and the patient experience. 


\section{Recurrent Aphthous Stomatitis: Diagnosis and Management in Primary Care}

Jillian Hudson, MD

Family Medicine Residency Program, Aurora St. Luke's Medical Center, Milwaukee, WI

\begin{abstract}
Recurrent aphthous stomatitis (canker sores) is a very common oral condition that remains incompletely understood. Presentation has been well-classified into minor, major or herpetiform subcategories based on clinical features, but exact etiology is unknown. Because etiology is unclear, treatments are primarily empiric and aimed at symptom reduction rather than prevention or cure. However, there are several methods, both topical and systemic, that can be easily and affordably utilized in the primary care setting. (J Patient-Centered Res Rev. 2014;1:197-200.)
\end{abstract}

\section{Keywords}

recurrent aphthous stomatitis, aphthous ulcer, treatment, management

\section{Introduction}

Recurrent aphthous stomatitis (RAS) is a painful disease of the oral mucosa that affects $20 \%$ of the general population worldwide and possibly more in the North American population. It is one of the most common oral complaints seen in the primary care setting. Despite its prevalence, its causes remain largely unknown. ${ }^{1}$ Because of this, finding a definitive treatment has thus far been elusive, and treatments are largely empiric and focused on short-term symptom management. It is the goal of this paper to discuss various treatment options, both symptomatic and preventive, currently being used or proposed that could be relevant for use in primary care.

\section{Clinical Features}

There appears to be no consistent definition for what qualifies as recurrent aphthous stomatitis; however, one group has proposed at least three attacks of oral ulcers, not in the same focal site, over a 3-year period. ${ }^{2}$ Aphthous ulcers have been categorized into three groups: minor, major and

Correspondence: Jillian Hudson, MD, Lakeshore Medical Clinic, 3611 S. Chicago Avenue, Suite 100, South Milwaukee, WI, 53172, Phone: 414-762-7270, Fax: 414-762-7864,

Email: jillian.huyck@aurora.org herpetiform. Table 1 lists the categories of aphthous ulcers and their respective features. Minor aphthae are by far the most common presentation of RAS, representing $80-85 \%$ of cases. ${ }^{1-4}$ Ulcers are up to $1 \mathrm{~cm}$ in size, shallow and generally last 10-14 days. They occur on the labial or buccal mucosa, soft palate, tongue or floor of the mouth. ${ }^{1-4}$ There may be a prodromal burning or stinging sensation prior to appearance of the lesions. Scar formation does not occur with healing of minor aphthae..$^{1-4}$

Table 1. Categories of aphthous ulcers

\begin{tabular}{lcccc}
\hline & \multicolumn{4}{c}{ Features at presentation } \\
\cline { 2 - 5 } & $\begin{array}{c}\text { Size } \\
(\mathbf{m m})\end{array}$ & $\begin{array}{c}\text { No. of ulcers in } \\
\text { an outbreak }\end{array}$ & $\begin{array}{c}\text { Duration } \\
\text { (days) }\end{array}$ & Scarring \\
\hline Minor ulcers & $5-10$ & Few & $10-14$ & No \\
Major ulcers & $>10$ & Few & $14+$ & Yes \\
$\begin{array}{c}\text { Herpetiform } \\
\text { ulcers }\end{array}$ & $<5$ & Many (5-100) & $10-14$ & No \\
\hline
\end{tabular}

Major aphthae are rare, representing approximately $10-15 \%$ of RAS cases. They are defined by ulcers larger than $1 \mathrm{~cm}$, are generally deeper and have a longer duration than minor aphthae. Major aphthae may form a scar with healing. ${ }^{1-4}$ Major aphthae can be seen with human immunodeficiency virus (HIV) infection, and HIV testing should be considered when major aphthae are present. ${ }^{3}$

The herpetiform presentation is also rare, representing $10 \%$ or less of all RAS cases, and is defined by outbreaks of numerous, small, vesicular lesions. Vesicles may coalesce into larger ulcers. Duration of outbreaks is similar to minor aphthae, and generally no scar is formed..$^{1,3,4}$

Benign RAS should not be associated with systemic symptoms such as fever, lymphadenopathy, gastrointestinal symptoms, or mucosal or skin lesions beyond the nonkeratinized oral mucosa. If other symptoms are present, evaluation for systemic disease such as Behçet's disease, inflammatory bowel disease, celiac disease, HIV, or other autoimmune or immunodeficiency disorders should be considered. ${ }^{3,4}$ 


\section{Etiology/Pathogenesis}

While there are clear clinical characteristics to aphthae, the exact etiology remains unknown. ${ }^{1,3}$ It may be that RAS is a manifestation of a group of separate disorders rather than a single disease, which could explain the difficulty in definitively determining the cause. Aphthous stomatitis has a clear genetic predisposition. More than $42 \%$ of patients with RAS will have at least one first-degree relative who is also affected, and there is a $90 \%$ chance of developing RAS if both parents are affected. ${ }^{3}$ Ulcers appear to be a cell-mediated immune response in which T-cells and tumor necrosis factor (TNF)- $\alpha$ are involved. TNF- $\alpha$ is a proinflammatory cytokine that recruits T-cells and other leukocytes to the lesion, creating a painful papule that then ulcerates before healing. ${ }^{5}$ Predisposing factors include local trauma, stress, certain foods, hormonal fluctuations related to the menstrual cycle, and tobacco smoking. ${ }^{1-5}$ Sodium lauryl sulfate-containing toothpaste also may trigger aphthae in certain individuals. ${ }^{6}$ In addition, deficiencies in iron, folic acid or vitamin $B_{12}$ have been correlated with aphthous ulcer outbreaks. ${ }^{6}$

\section{Management Options}

As previously stated, because the exact etiology is unknown, finding a definitive treatment has been difficult. Avoidance of triggers should be recommended to limit recurrence. Most treatments currently used are palliative, with several systemic and preventive treatments recently proposed. There is currently no curative treatment known. Table $2^{7-14}$ summarizes treatments discussed in this article.

\section{Topical Treatments}

Amlexanox paste: Amlexanox is an anti-inflammatory agent that is approved by the U.S. Food and Drug Administration for the treatment of aphthous ulcers. Four randomized, placebo-controlled, double-blind, multicenter trials studied a total of 1,335 subjects and found amlexanox both safe and effective. ${ }^{7}$ Participants applied a small amount of 5\% amlexanox paste to their ulcers 4 times a day until their ulcers healed or the study ended. Patients had reduced pain (by day 2-3) and increased healing (starting from day 3-4 of treatment) compared to no treatment or placebo. Amlexanox also may help prevent ulcer formation if applied during the prodromal symptom phase; $35 \%$ of patients who applied amlexanox paste to the area of symptoms developed an ulcer after 3 days versus $97 \%$ of patients who received no treatment. In regards to adverse events, rates were similar for amlexanox (2.1\%) versus placebo $(2.5 \%)$. The most common complaint was transient burning or stinging at the application site, which occurred in equal rates in both groups; likely this sensation was due to the alcohol in the paste or the course texture rather than the amlexanox itself.

Topical steroids: Topical steroids are frequently used to treat aphthous ulcers. A recent multicenter, double-blind, placebo-controlled trial tested a total of 240 patients for the safety and efficacy of dexamethasone ointment. ${ }^{8}$ In the study, patients either applied a $1 \mathrm{mg} / \mathrm{g}$ dexamethasone ointment or placebo ointment to their lesion 3 times daily after meals for 5 days. Size and pain level of ulcers before and after treatment were evaluated. In addition, serum levels of dexamethasone were tested in 89 randomly selected subjects. They found significant reduction in ulcer size of the treatment group compared to the control group at the end of treatment, with a healing ratio of $83.3 \%$ in treatment group vs. $54.7 \%$ for placebo. Pain levels also were significantly improved with steroid treatment compared to placebo. Serum dexamethasone measurements found no detectable level of dexamethasone in either group, meaning a serum concentration level less than $0.502 \mathrm{ng} / \mathrm{mL}$.

Silver nitrate cautery: Silver nitrate cautery has been used for many years to relieve pain related to aphthous ulcers. There has been concern related to the painfulness of the procedure and possible impedance of ulcer healing with this technique. Two recent studies with 97 and 65 patients, respectively, showed significant pain relief and increased rate of healing compared to placebo. ${ }^{9,10}$ In each study the lesions were first anesthetized with topical lidocaine prior to the procedure. Treatment groups were treated with one application of a silver nitrate stick gently touched to the base of ulcer until the ulcer became white. Patient mouths were rinsed with copious amounts of water for 5 minutes after the procedure. Placebo groups used the same method but with a placebo treatment stick. Both studies found improved pain levels and decreased healing time compared to placebo. No significant adverse events were documented.

\section{Systemic Treatments}

Therapies such as thalidomide, dapsone, levamisole, pentoxifylline or colchicine have proved effective in treating RAS, but significant side effects limit their use, particularly in the primary care setting. ${ }^{2,5,6}$ Other treatments have recently shown some hope in providing successful treatment while limiting side effects and cost, although the studies have been small. The following sections will review these recently studied treatments.

Montelukast: In a small, randomized, double-blind, placebo-controlled study, the efficacy of systemic prednisone 
and montelukast were compared. ${ }^{11}$ With 20 patients in each arm, patients were treated with either a prednisone taper, montelukast or placebo for a course of 2 months and followed for a further 2 months following the end of treatment. Prednisone provided faster resolution and greater pain relief (by day 2-3) than montelukast, but montelukast did provide more benefit than placebo (day 3-4 vs. day 4-5). Overall, the number of ulcers during treatment was reduced by $76 \%$ while on prednisone, $71 \%$ while on montelukast and only $5 \%$ for the placebo group. Prednisone had a greater rate of adverse events $(30 \%)$ versus montelukast $(10 \%)$ or placebo $(10 \%)$. This study was designed as a pilot study, and a larger study would be needed to confirm efficacy of montelukast as a long-term treatment.

Vitamin $B_{12}$ supplementation: One recent study tested the efficacy of vitamin $B_{12}$ supplement in reduction of aphthous ulcer outbreaks. In a randomized, double-blind, placebo-controlled test, a total of 58 patients were studied to determine the effect of $1,000 \mathrm{mcg}$ of sublingual vitamin $\mathrm{B}_{12}$ on aphthous ulcer outbreaks. ${ }^{12}$ The authors found that "the duration of outbreaks, the number of ulcers, and the level of pain were reduced significantly $(\mathrm{P}>0.05)$ at 5 and 6 months of treatment." In addition, they found that $74 \%$ of the treatment group reached had no ulcers by the end of their 6-month observation period vs. $32 \%$ in the control group. The primary limitation of the study was sample size, with only 31 patients in the treatment group and 27 in the control group. However, vitamin $\mathrm{B}_{12}$ is relatively safe and inexpensive, making it a reasonable option to trial in patients suffering from RAS.

Omega-3 fatty acids: A recently published study assessed the efficacy of dietary omega-3 fatty acid supplementation in reducing symptoms of RAS and improving quality of life. ${ }^{13}$ Fifty patients were divided evenly into a treatment group receiving $1,000 \mathrm{mg}$ of omega-3 supplementation daily versus a placebo group and were followed monthly for 6 months. Each omega-3 capsule provided $200 \mathrm{mg}$ of docosahexaenoic acid and $300 \mathrm{mg}$ of eicosapentaenoic acid. By 3 months of treatment, level of pain, number of ulcers and length of outbreaks were reduced with omega-3 supplementation versus placebo, and this effect lasted for the remainder of the observation period of 6 months. No adverse events were reported during the duration of the study.

Multivitamin supplementation of no benefit: Although certain vitamin deficiencies have been proposed as playing a role in RAS, a large study that followed 160 patients for 1 year showed no benefit in the use of a daily multivitamin (containing $100 \%$ of the recommended daily intake of essential vitamins) to reduce number of ulcers or frequency of outbreaks when compared to placebo. ${ }^{14}$ Multivitamin use should not be recommended to reduce ulcer outbreaks.

Table 2. Summary of treatments for recurrent aphthous stomatitis

\begin{tabular}{|c|c|c|c|}
\hline Treatment & Dosage & $\begin{array}{l}\text { No. of } \\
\text { patients } \\
\text { studied }\end{array}$ & Outcomes \\
\hline Amlexanox $5 \%$ paste $^{7}$ & Apply to ulcer 4 times daily & 1,335 & $\begin{array}{l}\text { Pain resolved by day } 3 \\
\text { Ulcer healed by day } 3 \\
\text { Ulcer prevention if started during prodromal stage }\end{array}$ \\
\hline $\begin{array}{l}\text { Dexamethasone } 0.1 \% \\
\text { ointment }^{8}\end{array}$ & $\begin{array}{l}\text { Apply to ulcer } 3 \text { times } \\
\text { daily after meals }\end{array}$ & 89 & Pain reduction \\
\hline Silver nitrate cautery ${ }^{9,10}$ & $\begin{array}{l}\text { One application to ulcer } \\
\text { until ulcer blanches }\end{array}$ & 162 & Pain reduction by day 1 \\
\hline Montelukast ${ }^{11}$ & 10 mg orally daily & 40 & $\begin{array}{l}\text { Resolution of pain in 3-4 days } \\
\text { Decreased number of ulcers during treatment }\end{array}$ \\
\hline Vitamin $\mathrm{B}_{12}{ }^{12}$ & 1,000 mcg sublingually daily & 58 & No ulcers after 6 months of treatment \\
\hline Omega-3 fatty acids ${ }^{13}$ & 200 mg DHA/300 mg EPA daily & 50 & $\begin{array}{l}\text { Decreased number of ulcers, decreased pain, } \\
\text { and outbreak duration shortened by } 3 \text { months }\end{array}$ \\
\hline Multivitamin ${ }^{14}$ & 100\% RDI daily & 160 & No benefit \\
\hline
\end{tabular}

$D H A$, docosahexaenoic acid; EPA, eicosapentaenoic acid; RDI, recommended daily intake. 


\section{Conclusions}

Recurrent aphthous stomatitis remains a poorly understood entity, despite having clear clinical features. However, it is a common oral complaint and causes significant pain and impact on quality of life. Much research has been done to find treatments to reduce pain related to, duration of and frequency of ulcer outbreaks. There are several treatment options, both local and systemic, that could be helpful for management of aphthae in the primary care setting. More research needs to be done regarding recent systemic treatments such as montelukast, vitamin $\mathrm{B}_{12}$ or omega-3 fatty acid supplementation to prove efficacy, but safety and relatively low cost make these reasonable options to trial if there is a possibility the treatment will improve symptoms in patients with frequent recurrence.

\section{Conflicts of Interest}

None.

\section{References}

1. Preeti L, Magesh K, Rajkumar K, Karthik R. Recurrent aphthous stomatitis. J Oral Maxillofac Pathol. 2011;15:252-6.

2. Natah SS, Konttinen YT, Enattah NS, Ashammakhi N, Sharkey KA, Häyrinen-Immonen R. Recurrent aphthous ulcers today: a review of growing knowledge. Int J Oral Maxillofac Surg. 2004;33:221-34.

3. Femiano F, Lanza A, Buonaiuto C, et al. Guidelines for diagnosis and management of aphthous stomatitis. Pediatr Infect Dis J. 2007;26:728-32.

4. McBride DR. Management of aphthous ulcers. Am Fam Physician. 2000;62:149-54, 160.
5. Scully C, Gorsky M, Lozada-Nur F. The diagnosis and management of recurrent aphthous stomatitis: a consensus approach. J Am Dent Assoc. 2003;134:200-7.

6. Jurge S, Kuffer R, Scully C, Porter SR. Mucosal disease series. Number VI. Recurrent aphthous stomatitis. Oral Dis. 2006;12:1-21.

7. Bell J. Amlexanox for the treatment of recurrent aphthous ulcers. Clin Drug Invest. 2005;25:555-66.

8. Liu C, Zhou Z, Liu G, et al. Efficacy and safety of dexamethasone ointment on recurrent aphthous ulceration. Am J Med. 2012;125: 292-301.

9. Alidaee MR, Taheri A, Mansoori P, Ghodsi SZ. Silver nitrate cautery in aphthous stomatitis: a randomized controlled trial. Br J Dermatol. 2005;153:521-5.

10. Soylu Özler G. Silver nitrate cauterization: a treatment option for aphthous stomatitis. J Craniomaxillofac Surg. 2014;42:e281-3.

11. Femiano F, Buonaiuto C, Gombos F, Lanza A, Cirillo N. Pilot study on recurrent aphthous stomatitis (RAS): a randomized placebocontrolled trial for the comparative therapeutic effects of systemic prednisone and systemic montelukast in subjects unresponsive to topical therapy. Oral Surg Oral Med Oral Pathol Oral Radiol Endod. 2010;109:402-7.

12. Volkov I, Rudoy I, Freud T, et al. Effectiveness of vitamin $B_{12}$ in treating recurrent aphthous stomatitis: a randomized, double-blind, placebo-controlled trial. J Am Board Fam Med. 2009;22:9-16.

13. El Khouli AM, El-Gendy EA. Efficacy of omega-3 in treatment of recurrent aphthous stomatitis and improvement of quality of life: a randomized, double-blind, placebo-controlled study. Oral Surg Oral Med Oral Pathol Oral Radiol. 2014;117:191-6.

14. Lalla RV, Choquette LE, Feinn RS, et al. Multivitamin therapy for recurrent aphthous stomatitis: a randomized, double-masked, placebo-controlled trial. J Am Dent Assoc. 2012;143:370-6.

(C) 2014 Aurora Health Care, Inc. 